\title{
REVIEW
}

\section{Sodium-Glucose Cotransporter-2 Inhibition and the Glomerulus: A Review}

\author{
Sanjay Kalra $\cdot$ Vikram Singh $\cdot$ Dinesh Nagrale
}

Received: April 28, 2016/Published online: July 16, 2016

(C) The Author(s) 2016. This article is published with open access at Springerlink.com

\begin{abstract}
Blood glucose-lowering treatment options generally target insulin action or beta-cell function. In diabetes, expression of the sodium-glucose cotransporter-2 (SGLT2) genes is up-regulated and renal threshold increased, resulting in increased glucose reabsorption from glomerular filtrate, reducing urinary glucose excretion and worsening the hyperglycemic condition. The SGLT2 inhibitors (SGLT2i) are a novel class of anti-diabetic drugs that lower blood glucose levels through the suppression of renal glucose reabsorption thereby promoting renal glucose excretion. The efficacy of SGLT2i is reduced in renal impairment because the ability of glucose-lowering is directly proportional to
\end{abstract}

Enhanced content To view enhanced content for this article go to http://www.medengine.com/Redeem/ 51E4F0605031B5AE. glomerular filtration rate. On the other hand, ongoing research suggests that SGLT2i may offer potential nephroprotection in diabetes. The SGLT2i have been shown to reduce glomerular hyperfiltration, systemic and intraglomerular pressure and the biochemical progression of chronic kidney disease. Additional mechanisms through which SGLT2i exert nephroprotection may include normalizing blood pressure and uricemia. This review explores this bidirectional relationship of the SGLT2i and the glomerulus. While SGLT2i exhibit reduced efficacy in later stages, they exhibit nephroprotective effects in early stages of renal impairment.

Funding: Janssen India (Pharmaceutical division of Johnson \& Johnson).

Keywords: Chronic kidney disease; Diabetes mellitus; Endocrinology; Glomerulus; Glomerular hyperfiltration; Nephrology; Nephroprotection; SGLT2 inhibitors

\section{INTRODUCTION}

Awareness on the role of kidney in glucose homeostasis has led to the development of a new 
pharmacological class of anti-hyperglycemic agents. Sodium-glucose cotransporter-2 inhibitors (SGLT2i) are a novel and growing class of anti-diabetic drugs that lower blood glucose levels through the inhibition of renal glucose reabsorption thereby enhancing renal glucose excretion [1-3]. This approach of glucose-lowering is independent of insulin action or beta-cell function and complementary to other anti-diabetic drugs [4]. Treatment with SGLT2i has demonstrated improvements in glycosylated hemoglobin (HbA1c), reduction in body weight and modest lowering of blood pressure (BP) $[2,5]$. The pharmacological inhibition of renal glucose reabsorption through selective inhibition of SGLT2 has thus opened a new arena in the treatment of diabetes mellitus (DM).

The ability of SGLT2i to reduce the plasma glucose levels is directly proportional to the glomerular filtration rate (GFR) [6], and hence, is expected to be decreased in chronic kidney disease (CKD). On the other hand, ongoing research suggests that SGLT2i may offer potential nephroprotection in diabetes [7-9]. The SGLT2i could reduce glomerular hyperfiltration, systemic and intraglomerular pressure and accordingly, the progression of CKD, even in those without DM [10]. The efficacy of SGLT2i decreases with renal impairment while on the other hand, SGLT2i protect against the renal injury. Thus, the SGLT2i and glomerulus both impact each other and possess a bidirectional relation. Exploring this bidirectional relationship of the SGLT2i and the glomerulus in healthy individuals, patients with diabetes, and in different degrees of kidney disease is of particular importance to understand their therapeutic potential in these conditions. This article is based on previously conducted studies and does not involve any new studies of human or animal subjects performed by any of the authors.

\section{ROLE OF KIDNEYS IN GLUCOSE METABOLISM}

The kidneys play a major role in the regulation of glucose reabsorption and in maintaining the overall metabolic balance in humans [6]. More than $99 \%$ of the plasma glucose that filters through the renal glomeruli is reabsorbed [11]. The transport of glucose across cell membranes is mediated primarily by two different types of sodium-glucose cotransporters: SGLT1 and SGLT2. The SGLT2, a high-capacity, low-affinity glucose transporter is located primarily in the brush border membrane of the $\mathrm{S} 1$ segment of the proximal renal tubule and is responsible for reabsorption of more than $90 \%$ of filtered glucose in the kidney [12]. The SGLT1, a high-affinity, low-capacity glucose transporter, reabsorbs the remaining $10 \%$ of filtered glucose and is primarily expressed in the gastrointestinal tract (Fig. 1) [13].

\section{Glucose Reabsorption in Healthy Kidney}

Kidneys achieve glucose homeostasis via three pathways: uptake of glucose from the circulation, renal gluconeogenesis and glucose reabsorption from the glomerular filtrate [6]. In a healthy individual, about $180 \mathrm{~g}$ of glucose is filtered from plasma by glomeruli every day. However, under normal circumstances, the filtered glucose is almost completely reabsorbed in the proximal tubules by the SGLT2 and SGLT1, leaving minimal or no glucose in the urine [6]. This state of no or minimal glucosuria is maintained in the healthy individuals (Fig. 1).

This ability of proximal tubules to reabsorb almost all the filtered glucose, via SGLTs, linearly increases with the increase in filtered glucose load until the maximum glucose 


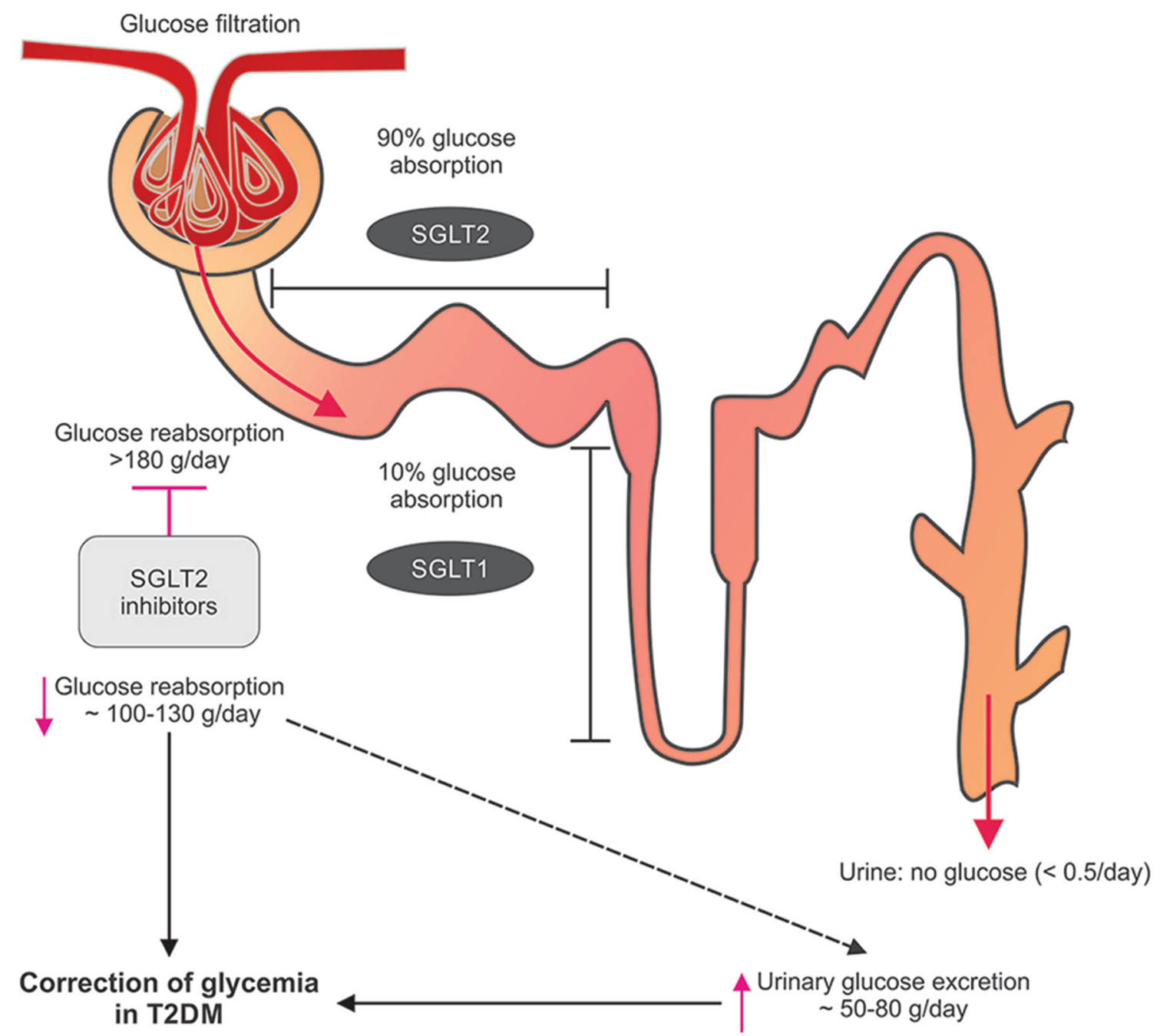

Fig. 1 Glucose reabsorption pathway and SGLT2 inhibition. T2DM type 2 diabetes mellitus, SGLT sodium-glucose cotransporter

transport capacity (known as Tm glucose) is reached. The filtered glucose load is a result of plasma glucose concentration and the GFR. The concentration at which the plasma glucose levels reach the $\mathrm{Tm}$ glucose is called renal threshold for glucose excretion. It equates the GFR of $260-350 \mathrm{mg} / \mathrm{min} / 1.73 \mathrm{~m}^{2}$, which is equivalent to plasma glucose concentrations of approximately $200 \mathrm{mg} / \mathrm{dL} \quad(11.0 \mathrm{mmol} / \mathrm{L})$ in healthy adults. Above this concentration, the excess glucose cannot be reabsorbed and is excreted resulting in glucosuria $[6,14]$.

\section{Glucose Reabsorption in Diabetic Kidney}

In patients with diabetes, the blood glucose levels increase and exceed the Tm glucose at a threshold of approximately $200 \mathrm{mg} / \mathrm{dL}$. Consequently, the excess glucose is not reabsorbed leading to glucosuria. However, in diabetes, expression of the SGLT2 transporter genes is up-regulated and the renal threshold is increased [6, 15]. This results in increased glucose reabsorption from glomerular filtrate in patients with diabetes reducing urinary 
glucose excretion (UGE) and further worsening the hyperglycemic condition $[6,15]$. Hence, suppressing the glucose reabsorption and increasing the glucosuria through inhibition of the SGLT2 is considered as an effective and practical approach for the correction of this intensified hyperglycemia.

\section{EFFECTS OF SGLT2 INHIBITORS}

\section{In Healthy Individuals}

Clinical studies have revealed that treatment with SGLT2i reduces renal threshold for glucose excretion and increases UGE in healthy individuals, dose-dependently $[3,16,17]$. Existing evidence suggests that treatment with SGLT2i in healthy individuals leads to continuous glucose excretion in urine with a corresponding loss in calories [3, 16, 17]. However, this increase in glucose excretion does not alter the plasma glucose levels in healthy individuals since liver compensates the loss of glucose with increased glucose production to avoid hypoglycemia. This raises the possibility of SGLT2i being used for weight reduction among healthy individuals. Empagliflozin has demonstrated significantly increased glucose excretion in urine as well as weight loss without any changes in body water or protein content in diet-induced obese rats for 4 weeks [18]. However, evidence confirming the role of SGLT2i as an option for weight reduction treatment in healthy humans is limited. In a recent 12-week, placebo-controlled, dose-ranging study, canagliflozin $(50,100$, or $300 \mathrm{mg} /$ day) reduced body weight by $1-2 \%$, as compared with placebo. Though the drug was generally well-tolerated in overweight and obese participants without DM, the magnitude of weight reduction did not support the use of
SGLT2 inhibition monotherapy as a viable weight loss intervention [19].

\section{In Patients with Diabetes}

SGLT2i inhibit renal glucose reabsorption by inhibiting SGLT2 in the kidney, which in turn facilitates the renal glucose excretion (Fig. 1). As the UGE increases, the plasma glucose levels fall leading to the improvement in all glycemic parameters [1-3]. Selective SGLT2i exhibit minimal or no risk of hypoglycemia as their action diminishes when the plasma glucose concentrations decrease [20]. The SGLT2i are generally well-tolerated with common adverse events of urinary and genital infections [21]. An added advantage is that SGLT2i can be administered as an add-on therapy along with the existing anti-diabetic agents because of their insulin-independent mechanism of action.

Many selective SGLT2i have been developed and/or are currently under development. Canagliflozin, dapagliflozin and empagliflozin have now been approved for clinical use in patients with type $2 \mathrm{DM}$ (T2DM) in the United States, Europe, and other countries including India [22]. Ipragliflozin has been approved and marketed in Japan [23]. Other SGLT2i like luseogliflozin, tofogliflozin, ertugliflozin, and sotagliflozin are in the late phase of clinical development [24, 25].

Several large randomized clinical studies have been conducted to explore the effects of SGLT2i in patients with diabetes. These studies consistently confirmed that SGLT2i reduce hyperglycemia and improve glycemic control with associated weight loss due to increased UGE, and reduction in BP, when given as monotherapy or as an add-on treatment $[5,21,24,26-30]$. 
EFFECT OF GLOMERULAR HEALTH, AS MEASURED BY GFR, ON THE EFFICACY OF SGLT2 INHIBITORS

As the ability of SGLT2i to increase the UGE primarily depends on the GFR, it is observed that their pharmacodynamic response gradually decreases with increasing severity of renal dysfunction [28, 29, 31]. Clinical studies evaluating the efficacy of SGLT2i in patients with varying degrees of renal impairment suggest that the glucose-lowering capacity of the SGLT2i, as assessed by a reduction in HbA1c, is maintained in patients with mild renal impairment (stage $2 \mathrm{CKD}$; estimated glomerular filtration rate [eGFR] $60-90 \mathrm{~mL} /$ $\mathrm{min} / 1.73 \mathrm{~m}^{2}$ ). The glucose-lowering capacity decreases in patients with moderate renal impairment (stage 3 CKD: eGFR $\geq 30$ to $<60 \mathrm{~mL} / \mathrm{min} / 1.73 \mathrm{~m}^{2}$ ) and is limited in patients with severe renal impairment (stage 4 CKD: eGFR $<30 \mathrm{~mL} / \mathrm{min} / 1.73 \mathrm{~m}^{2}$ ), or in renal failure, with no glycemic therapeutic benefits [32-35]. The approved usage of various SGLT2i in different stages of CKD is summarized in Table 1.

\section{Mild Renal Impairment}

Evidences suggest that the glucose-lowering efficacy and safety of SGLT2i in patients with mild renal impairment was comparable to those observed in patients with normal kidney function [32, 34]. This implies that SGLT2i may be used in patients with mild renal impairment without dose-adjustments.

\section{Moderate Renal Impairment}

The ability of SGLT2i to promote UGE reduces with decreasing GFR, and hence, patients with moderate renal impairment have reduced glucose clearance than those with normal renal function or mild impairment. Many studies have noted significant increase in UGE in patients with moderate renal impairment (although less compared with normal or mildly impaired renal function, as expected) along with body weight reduction [32, 34-36]. This suggests that SGLT2i may be beneficial in patients with moderate renal impairment since the available oral anti-diabetic medications in these populations are limited. However, prescribing information should be referred regarding any restrictions or dosage adjustments for each SGLT2i.

\section{Severe Renal Impairment}

As the pharmacodynamic effect of SGLT2i is primarily dependent on GFR, no glycemic therapeutic benefits are anticipated in patients with severe renal impairment or renal failure. Evidence suggests little or no effect of SGLT2i on the UGE in patients with severe renal impairment or renal failure; hence, its use is contraindicated in this population [32, 33, 35].

\section{PATHOGENESIS OF DIABETIC NEPHROPATHY}

Of the known complications of diabetes, diabetic nephropathy (DN) is of particular concern as it is a leading cause of CKD, worldwide $[8,9,37]$. DN is characterized by a progressive increase in proteinuria and decline in GFR, associated with hypertension, finally culminating in end-stage renal disease (ESRD) $[38,39]$. Recent research has been focused on understanding the pathogenic mechanisms that initiate the progression of early DN towards ESRD. 
Table 1 Sodium-glucose cotransporter-2 inhibitors in different stages of kidney disease [81-84]

\begin{tabular}{|c|c|c|c|c|c|}
\hline $\begin{array}{l}\text { Stage } \\
\text { of } \\
\text { CKD }\end{array}$ & Description & $\begin{array}{l}\text { GFR }(\mathrm{mL} / \mathrm{min} / \\
\left.1.73 \mathrm{~m}^{2}\right)\end{array}$ & Canagliflozin & Dapagliflozin & Empagliflozin \\
\hline 1 & $\begin{array}{l}\text { Kidney damage with normal kidney } \\
\text { function }\end{array}$ & $\geq 90$ & $\sqrt{ }$ & $\sqrt{ }$ & $\sqrt{ }$ \\
\hline 2 & $\begin{array}{l}\text { Kidney damage with mild loss of kidney } \\
\text { function }\end{array}$ & $89-60$ & $\sqrt{ }$ & $\sqrt{ }$ & $\sqrt{ }$ \\
\hline $3 a$ & Mild to moderate loss of kidney function & $59-45$ & $\sqrt{ }^{a}$ & $\mathrm{X}$ & $\sqrt{ }$ \\
\hline $3 b$ & Moderate to severe loss of kidney function & $44-30$ & $\mathrm{X}$ & $\mathrm{X}$ & $\mathrm{X}$ \\
\hline 4 & Severe loss of kidney function & $29-15$ & $\mathrm{X}$ & $\mathrm{X}$ & $\mathrm{X}$ \\
\hline 5 & Kidney failure & $<15$ & $\mathrm{X}$ & $\mathrm{X}$ & $\mathrm{X}$ \\
\hline
\end{tabular}

Approved doses:

Canagliflozin-The recommended dose is $100 \mathrm{mg}$ or $300 \mathrm{mg} /$ day. The $300 \mathrm{mg}$ dose may be considered for patients tolerating $100 \mathrm{mg} /$ day who have an estimated GFR of $60 \mathrm{~mL} / \mathrm{min} / 1.73 \mathrm{~m}^{2}$ or greater and require additional glycemic control

Dapagliflozin-recommended starting dose is $5 \mathrm{mg}$, can be increased to $10 \mathrm{mg} /$ day in patients tolerating $5 \mathrm{mg} /$ day who require additional glycemic control

Empagliflozin-recommended starting dose is $10 \mathrm{mg}$, can be increased to $25 \mathrm{mg} /$ day

$\sqrt{ }=$ Can be used, $\mathrm{X}=$ cannot be used

CKD chronic kidney disease, GFR glomerular filtration rate

${ }^{a}$ Limited to $100 \mathrm{mg} /$ day

\section{Glomerular Hyperfiltration}

Glomerular hyperfiltration is an early renal hemodynamic abnormality in patients with diabetes and has been identified as a surrogate marker of increased intraglomerular pressure [40]. Its prevalence in type $1 \mathrm{DM}$ (T1DM) and T2DM has been reported to be as high as 50\% [41]. It is defined by a GFR of $125-140 \mathrm{~mL} /$ $\min / 1.73 \mathrm{~m}^{2}$, or $>2$ standard deviations above the mean GFR in healthy individuals [42]. Hyperfiltration is also observed in pre-diabetic states such as impaired fasting glycemia [43] and is a risk factor of DN [44]. The pathogenesis of glomerular hyperfiltration is not completely understood; however, both hemodynamic and tubular mechanisms have been associated with this condition (Fig. 2).

\section{Hemodynamic Hypothesis}

The hemodynamic hypothesis is based on the changes in afferent and efferent arteriolar resistances in the glomerulus, primarily due to activation of renin-angiotensin-aldosterone system (RAAS), leading to afferent arteriolar vasodilatation and efferent arteriolar vasoconstriction and consequent hyperfiltration $[42,44]$. Therapies targeting the reduction in intraglomerular pressure by blocking the RAAS have been used in patients with DN over decades. However, studies reporting the effect of RAAS inhibitors on progression of renal disease have resulted in conflicting results. Many studies have shown that the RAAS blockage monotherapies including the angiotensin-converting enzyme inhibitors (ACEi) have not been able to fully arrest the 


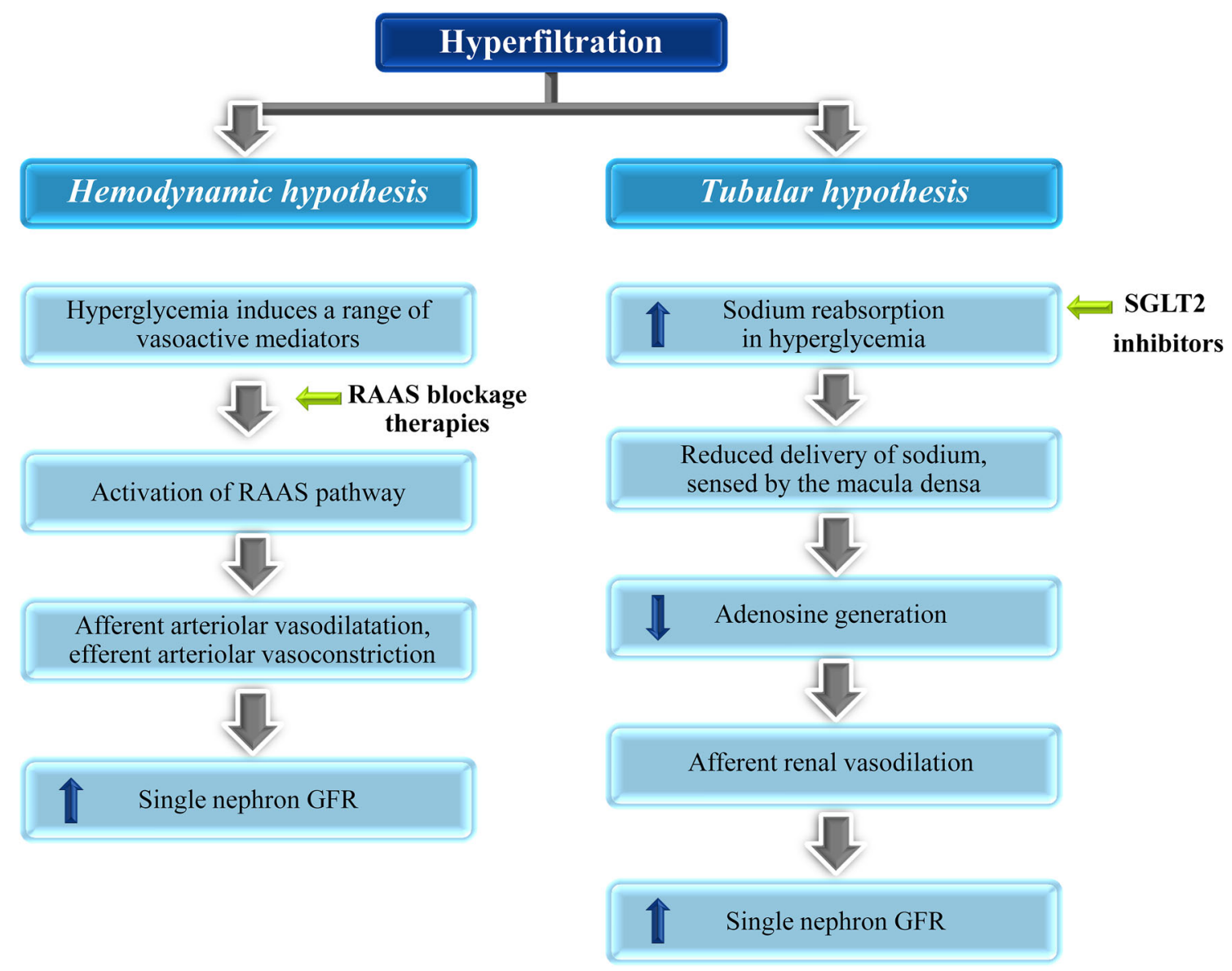

Fig. 2 Pathogenesis of glomerular hyperfiltration and mode of action of nephroprotective drugs. GFR glomerular filtration rate, $R A A S$ renin-angiotensin-aldosterone system, SGLT2 sodium-glucose cotransporter-2

progression of disease in diabetic patients [45-48]. Even the dual RAAS blockage mechanism involving both ACE inhibition and renin inhibition was not able to fully attenuate hyperfiltration [7]. Hence, as the nephroprotective effects of RAAS blockage therapies are limited, there is a crucial need for alternative therapies for renal protection in patients with diabetes [7].

\section{Tubular Hypothesis}

The tubular hypothesis is based on the increase in sodium reabsorption at the proximal tubule mediated by SGLT2. In diabetes, hyperglycemia leads to filtered glucose load coupled with increased expression of SGLT2. As a result, more glucose and sodium is reabsorbed in the proximal tubules leading to reduced delivery of sodium to the macula densa. This condition is sensed by the macula densa as a reduction in effective circulating volume causing reduction in adenosine generation in the juxtaglomerular apparatus. This causes afferent renal vasodilation leading to hyperfiltration (Fig. 3) [10]. The need for alternative non-RAAS dependent therapies for DN has highlighted the importance of therapies targeting tubular hypothesis.

\section{Hypertension}

In T2DM, hypertension commonly precedes kidney disease [39]. Several studies have 


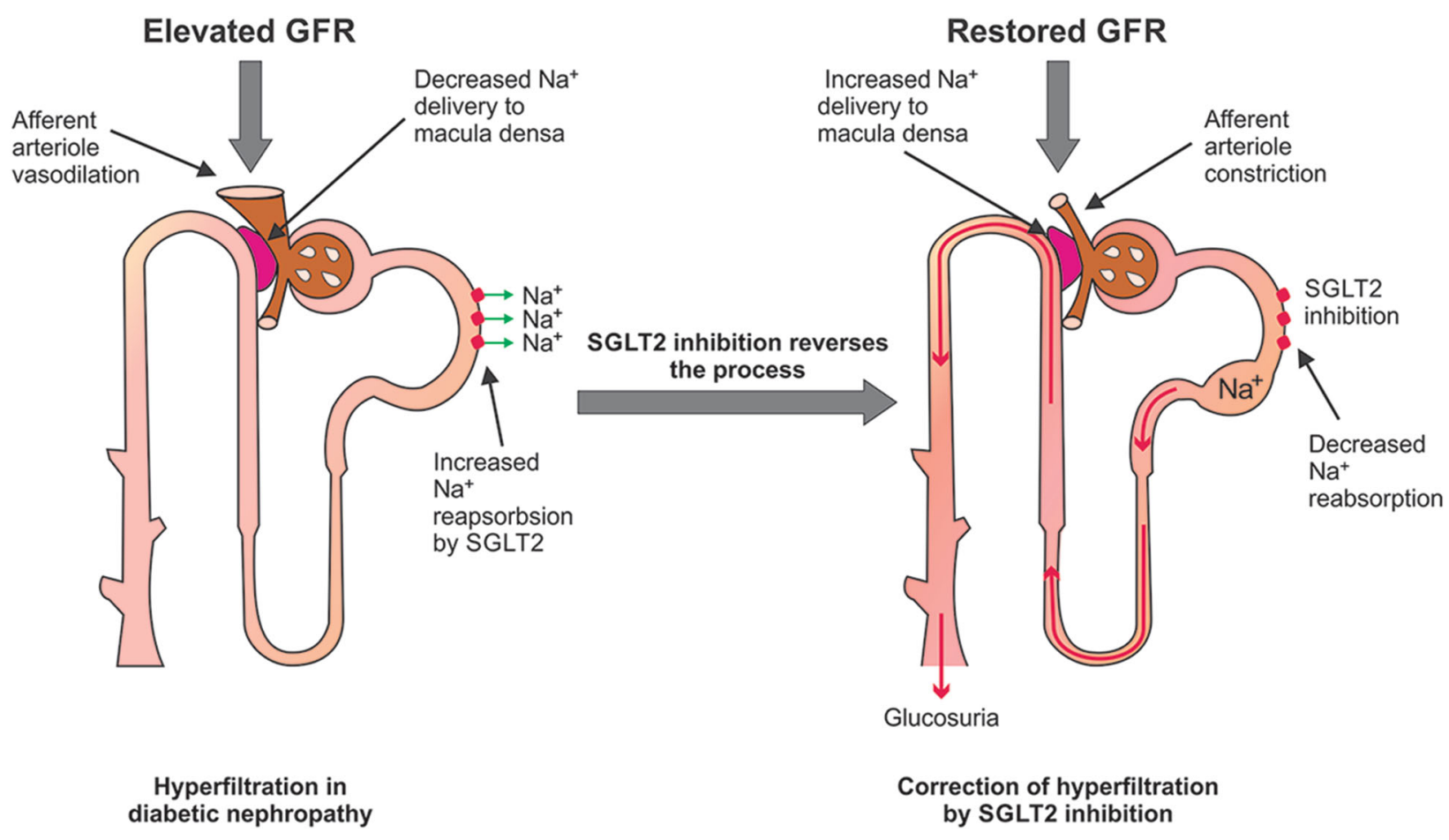

Fig. 3 Hyperfiltration in diabetic nephropathy and reduction of hyperfiltration by SGLT2 inhibitors. GFR glomerular filtration rate $T 2 D M$ type 2 diabetes mellitus, $S G L T 2$ sodium-glucose cotransporter-2

demonstrated that 58-70\% newly diagnosed patients with T2DM already had hypertension $[49,50]$. There is a multifactorial etiology of development of hypertension in diabetes and the hypertension thus developed, contributes to DN via mechanisms mediated by common factors. The major factors contributing to hypertension include volume expansion due to increased sodium reabsorption and peripheral vasoconstriction. Further, activation of the RAAS, upregulation of endothelin1 and reactive oxygen species and downregulation of nitric oxide contribute to this process [39]. A tight control of BP is apparently the most important single intervention to decrease progressive nephropathy in patients with diabetes. Treatment of hypertension improves albuminuria and results in delayed onset and progression to DN [38]. Currently, RAAS inhibitors such as ACE inhibitors and angiotensin receptor blockers (ARBs) are considered as first-line of treatment for hypertension in patients with diabetes [51].

\section{EFFECT OF SGLT2 INHIBITORS ON RENAL FUNCTION}

The mechanism of action of SGLT2i depends on filtration of glucose at the glomerulus. Therefore, these drugs are less effective in patients with renal impairment. However, evidence suggests that SGLT2i may possess nephroprotective effect in DN. The SGLT2i have been shown to reduce glomerular hyperfiltration, systemic and intraglomerular pressure, and accordingly, the progression of CKD [8, 52].

The rationale for using SGLT2i as a nephroprotective therapy is compelling as SGLT2 $\mathrm{i}$ has the potential to modify the 
Table 2 Potential mechanisms of nephroprotection by SGLT2 inhibitors

\begin{tabular}{ll}
\hline Mechanism & Effects of SGLT2 inhibitor \\
\hline Metabolic & \\
& HbAlc $\downarrow$ \\
& Weight $\downarrow$ \\
& Serum uric acid $\downarrow$ \\
& Blood pressure $\downarrow$ \\
Renal & \\
Glomerular & Inhibition of tubuloglomerular feedback \\
& Glomerular hyperfiltration $\downarrow$ \\
& Albuminuria $\downarrow$ \\
Tubular & Tubular apoptosis $\downarrow$ \\
& Proximal tubule $\mathrm{Na}^{+}$reabsorption $\downarrow$ \\
General & Intracellular oxidative stress $\downarrow$ \\
\hline
\end{tabular}

$\mathrm{HbAlc}$ glycosylated hemoglobin, $\mathrm{Na}$ sodium, SGLT2 sodium-glucose cotransporter-2

pathophysiologic process suggested in the tubular hypothesis. These inhibitors increase the sodium delivery to the distal convoluted tubule thereby increasing intracellular adenosine, causing afferent arteriolar vasoconstriction, which in turn leads to reduced glomerular pressure, hyperfiltration and renal injury (Fig. 3) [37]. This mechanism of preservation of renal function by SGLT2i is complementary to that of other nephroprotective therapies including those targeting RAAS. The mechanisms through which SGLT2i exert nephroprotection are summarized in Table 2.

Experimental studies have suggested that SGLT2i ameliorate glomerular hyperfiltration reduce albuminuria, kidney hypertrophy and inflammation in animal models of T1DM and T2DM [8, 10, 53]. Treatment with SGLT2i has shown to reduce intrarenal inflammation in diabetic $\mathrm{db} / \mathrm{db}$ mice [52]. In this study, decreased macrophage infiltration, inflammatory response and oxidative stress in the kidney were noted with dapagliflozin treatment. These effects were found to be independent of it's glucose-lowering effects. Conversely, a recent study in T1DM endothelial nitric oxide synthase knockout mice, showed no renoprotective benefits of empagliflozin apart from glucose-lowering effects [54].

Preclinical data on nephroprotective role of SGLT2i have been supported by various clinical studies. Effect of empagliflozin (25 mg once daily) on the glomerular hyperfiltration was studied in patients with T1DM, with or without hyperfiltration, in an eight-week open-label, stratified clinical study. Treatment with empagliflozin attenuated the hyperfiltration by $-33 \mathrm{~mL} / \mathrm{min} / 1.73 \mathrm{~m}^{2}$ in patients with baseline hyperfiltration in clamped euglycemic conditions and by $44 \mathrm{~mL} / \mathrm{min} / 1.73 \mathrm{~m}^{2}$ in hyperglycemic conditions. This change in GFR occurred in conjunction with significant decrease in renal blood flow and systolic BP and an increase in renal vascular resistance confirming the role of tubular hypothesis in the pathogenesis of glomerular hyperfiltration [37]. The nephroprotective role of SGLT2i is yet to be confirmed and there is a need for exclusive studies on nephroprotection. One such clinical outcome study, the Canagliflozin and Renal Events in Diabetes with Established Nephropathy Clinical Evaluation (CREDENCE; ClinicalTrials.gov identifier: NCT02065791) study, is in progress [55].

Besides having anti-hyperglycemic activity, SGLT2i induce weight loss, osmotic diuresis and BP-lowering. Although not approved for BP-lowering, they can potentially help achieve target BP in patients with diabetes who require $\mathrm{BP}$ reduction of $7-10 \mathrm{mmHg}[56,57]$. While the exact mechanism of BP-lowering effect with SGLT2i is not yet fully established, the osmotic diuretic effect of these agents has been related 
most often to BP reduction [56]. A randomized, placebo-controlled study in patients with T2DM $(n=75)$ showed reductions in 24-h systolic BP $(-3.3 \mathrm{mmHg})$ with dapagliflozin treatment (10 mg/day) compared with $-0.9 \mathrm{mmHg}$ for placebo [58]. A similar reduction with dapagliflozin (10 mg/day) in seated systolic BP [-4.28 (95\% confidence interval: $-6.54,-2.02)$ ] compared with placebo was noted in a recent 12-week, phase 3 study involving 449 patients with T2DM and hypertension. The BP-lowering effect observed with dapagliflozin was attributed to the possible diuretic effect [57]. Similar results were found in another 26-week, phase 3 study that evaluated the efficacy and safety of two doses of canagliflozin in patients with uncontrolled T2DM and stage 3 CKD $(n=269)$. Both the doses (100 and $300 \mathrm{mg}$ ) of canagliflozin showed reductions from baseline in systolic BP $(-6.1$ and $-6.4 \mathrm{mmHg}$, respectively) and diastolic BP (-2.6 and $-3.5 \mathrm{mmHg}$, respectively) compared with placebo. The reduction in BP may be attributed to the reduced fluid volume secondary to osmotic diuretic property of SGLT2i [36].

SGLT2i reduces albuminuria, which is a predominant renal risk marker in DN [59]. Although the exact mechanism of albuminuria-lowering effect of SGLT2i is not yet elucidated, it seems to be independent of changes in eGFR, systolic BP, body weight, or HbA1c [60]. The albuminuria-lowering properties of SGLT2i have been demonstrated in various studies $[36,60,61]$. In patients with T2DM and stage $3 \mathrm{CKD}$, canagliflozin $100 \mathrm{mg} /$ day has been shown to reduce albuminuria by approximately $22 \%$ [36], relative to placebo. Similarly, empagliflozin $25 \mathrm{mg} /$ day decreased albuminuria by approximately $\quad 35 \%$ in another placebo-controlled study in patients with T2DM and CKD [61]. Another recent study in patients with diabetes and hypertension receiving RAAS blockade therapy showed reductions of approximately $35 \%$ with dapagliflozin $10 \mathrm{mg} /$ day, relative to placebo. In addition, these reductions were shown to be independent of changes in $\mathrm{HbA1c}$, systolic BP, body weight or eGFR [60].

Serum uric acid-lowering is another mechanism by which SGLT2i may exert their nephroprotective effect. Elevated levels of uric acid, also known as hyperuricemia, have been shown to highly correlate with the risk of renal damage in diabetes [62-64], and are responsible for microvascular disease in diabetes $[65,66]$. Animal studies have revealed that uric acid may induce tubular damage in DN [67]. Given the various metabolic effects of hyperuricemia in patients with diabetes, the uric acid-lowering effect of SGLT2i may be clinically important and is evidenced in several studies [27, 68, 69].

Treatment with SGLT2i has been shown to associate with an initial reduction in GFR, which stabilizes or attenuates towards baseline levels over time. In a pooled analysis of canagliflozin phase 3 study results, an initial reduction in eGFR was observed in T2DM patients treated with canagliflozin 100 and $300 \mathrm{mg}$ [70]. In another 26 week, phase-3 study in patients with T2DM with stage 3 CKD, at week 3, greater reductions in eGFR with canagliflozin were observed, which trended back towards baseline over the 26-week treatment. These transient changes in renal function with SGLT2i may not be considered harmful, but on the other hand may be indicative of underlying renal benefit and could be attributed to plasma volume reduction due to osmotic diuretic effect of SGLT2i [36]. In a long-term study, patients 
with T2DM inadequately controlled with metformin were randomized to receive 100 and $300 \mathrm{mg}$ of canagliflozin or glimepiride. The initial decreases in eGFR that occurred early after initiation of therapy were subsequently attenuated and remained stable over 104 weeks with both canagliflozin doses, unlike the progressive decline in GFR throughout the treatment period observed with glimepiride treatment [71].

\section{ADVANCED GLYCATION END PRODUCTS}

Advanced glycation end products (AGEs) are the covalent adducts of glucose with the plasma proteins, formed during chronic hyperglycemia. They cross-link with endogenous key molecules and support the development of DN [72]. Glycation of proteins disturb their normal function by altering conformation and enzymatic activity reduces degradation capacity and disrupts receptor recognition [73]. Interaction of AGEs with plasma membrane localized receptors named receptor for AGEs (RAGE) may lead to alteration in drug binding in the plasma, activation of platelets, generation of oxygen free radicals, impairment in fibrinolysis and impairment in immune system regulation resulting in the development and progression of various diabetic complications such as nephropathy, retinopathy and neuropathy [74]. Increased glucose uptake into proximal tubular cells via SGLT2 stimulates oxidative stress generation and RAGE expression. The AGEs-RAGE system has been shown to induce apoptosis of tubular cells, and thereby contribute to the progression of $\mathrm{DN}$.

Blockade of increased glucose uptake into renal proximal tubular cells by SGLT2 could be a therapeutic target for preventing AGEs-elicited tubular apoptosis and atrophy in DN. A previous study, aimed at understanding the process of augmentation of AGEs-elicited apoptosis of proximal tubular cells in vitro by SGLT2 inhibition, showed that complete blockade of SGLT2 expression and glucose entry into the cells resulted in augmentation of AGEs-induced apoptotic cell death of tubular cells via RAGE induction and increased reactive oxygen species generation [75]. Hence, SGLT2i could also exert their nephroprotective action by preventing of tubular apoptosis in $\mathrm{DN}$.

\section{SGLT2 INHIBITORS VERSUS RAAS-BASED AGENTS IN DIABETES THERAPY}

SGLT2i and the RAAS-based agents like ACEi or ARBs clearly have different mechanisms of action and different effects on the renal system (Table 3; Fig. 2). Considering their complementary mechanisms on the kidneys, theoretically, SGLT2i and ACEi/ARBs should have synergistic action. Indeed, in a recent experimental study, the combination of RAAS blockers and SGLT2i was associated with additive nephroprotective effect in DN compared to either drug alone [76].

The EMPA-REG OUTCOME study (ClinicalTrials.gov identifier: NCT01131676), assessing the long-term cardiovascular safety of empagliflozin in 7034 patients with T2DM and high cardiovascular risk, had $80 \%$ of the patients on RAAS blockers at baseline for BP reduction [77]. In addition, $26 \%$ of the patients had moderate renal impairment. Treatment with empagliflozin was associated with substantial improvement in all the renal function parameters. The nephroprotective effect of SGLT2i could also contribute to cardiovascular benefits by inducing volume depletion and neurohormonal activation [78]. 
Table 3 Mechanism of nephroprotection by SGLT2 inhibitors and RAAS-based agents

\begin{tabular}{|c|c|c|c|}
\hline \multirow[t]{2}{*}{ Nephroprotective effects } & \multirow{2}{*}{$\begin{array}{l}\text { SGLT2-based agents } \\
\text { SGLT2 inhibitor }\end{array}$} & \multicolumn{2}{|c|}{ RAAS-based agents } \\
\hline & & ACE inhibitor & ARB \\
\hline \multicolumn{4}{|l|}{ Metabolic effects } \\
\hline HbAlc & Reduced & & \\
\hline Body weight & Reduced & & \\
\hline Serum uric acid & Reduced & & \\
\hline \multicolumn{4}{|l|}{ Renal effects } \\
\hline \multicolumn{4}{|l|}{ Glomerular } \\
\hline Glucose reabsorption & Inhibited & & \\
\hline Glomerular hyperfiltration & Reduced & & \\
\hline Systemic and intraglomerular pressure & Reduced & Reduced & Reduced \\
\hline Albuminuria & Reduced & Reduced & Reduced \\
\hline \multicolumn{4}{|l|}{ Tubular } \\
\hline Proximal $\mathrm{Na}+$ reabsorption & Reduced & & \\
\hline Tubular inflammation and fibrosis & Reduced & & \\
\hline Tubular apoptosis & Reduced & & \\
\hline \multicolumn{4}{|l|}{ Glomerular and tubular } \\
\hline Tubule-glomerular feedback & Inhibited & & \\
\hline Glomerular and tubulointerstitial damage & & & Reduced \\
\hline \multicolumn{4}{|l|}{ Effects on RAAS } \\
\hline Production of angiotensin II & & Reduced & Reduced \\
\hline Secretion of aldosterone & & Reduced & \\
\hline Sodium and water retention & & Reduced & \\
\hline Peripheral vascular resistance & & Reduced & \\
\hline Bradykinin activation & & Inhibited & \\
\hline \multicolumn{4}{|l|}{ Other effects } \\
\hline Chronic hypoxia & & & Corrected \\
\hline Abnormal iron deposition in the interstitium & & & Prevented \\
\hline Plasminogen activator inhibitor-I & & & Inhibited \\
\hline Formation of advanced glycation end products & & & Inhibited \\
\hline Hydroxyl radical scavenging & & & Increased \\
\hline Expression of hemoxygenase- 1 and NADPH oxidase & & & Reduced \\
\hline Inflammatory cell infiltration & & & Ameliorated \\
\hline
\end{tabular}

\section{SAFETY OF SGLT2 INHIBITORS}

Although owing to their mechanism of action, SGLT2i are associated with low risk of hypoglycemia, they may lead to adverse effects like genital mycotic and urinary tract infections [21]. This may be attributed to the increased and prolonged glucosuria induced by SGLT2i. Although these side effects are not of serious nature, patients and the 
health care providers should be aware of them.

Another reported adverse effect of SGLT2i treatment is osmotic diuresis which results in dehydration, hypotension and renal impairment. Intravascular volume depletion was also observed with SGLT2i therapy [79]. In diabetic patients with renal impairment, a dose-dependent increase in serum creatinine, a known marker of renal damage, was observed [4]. A recent case report suggested the association of SGLT2i treatment with renal tubule dysfunction or Fanconi syndrome. However, it must be noted that this was observed in a T1DM patient, in whom canagliflozin was used off-label. Such potential adverse effects should be confirmed with further studies [80]. Hence, despite their proven nephroprotective role, SGLT2i should be prescribed with caution in patients with diabetes with significant renal impairment.

\section{CONCLUSIONS}

The SGLT2i with their novel insulin-independent glucose-lowering action and associated benefits on body weight and BP, are proving to be promising options either as monotherapy or as an add-on therapy for patients with diabetes not controlled with existing medications. Owing to their kidney-dependent mechanism of action, the glucose-lowering ability of SGLT2i decreases with an increase in severity of glomerular dysfunction in patients with DN. However, they have been proven to be useful at all stages of kidney disease except for the severe renal impairment or renal failure where they provide no therapeutic benefits. Additionally, in light of the recent research demonstrating the nephroprotective role of SGLT2i, they may prove to be beneficial for patients with $\mathrm{DN}$.

\section{ACKNOWLEDGMENTS}

The preparation of this review was supported by Janssen (Pharmaceutical division of Johnson \& Johnson), Mumbai, India. We acknowledge Dr. Himabindu Gutha for providing writing assistance and Dr. Madhavi Patil (both SIRO Clinpharm Pvt. Ltd) for additional editorial support for the development of this manuscript. This support was funded by Janssen, India. All authors meet International Committee of Medical Journal Editors (ICMJE) criteria and all those who fulfilled those criteria are listed as authors. All authors had access to the study data, provided direction and comments on the manuscript, made the final decision about where to publish these data and approved submission to this journal.

Disclosures. Dinesh Nagrale and Vikram Singh are employees and/or shareholders of Janssen, India. Sanjay Kalra has received honoraria from Johnson \& Johnson, Astra Zeneca and Boehringer Ingelheim for speaking assignments or advisory boards. All authors contributed to the development and review of this manuscript and confirm that they have read the Journal's position on issues involved in ethical publication and affirm that this report is consistent with those guidelines.

Compliance with Ethics Guidelines. This article is based on previously conducted studies and does not involve any new studies of human or animal subjects performed by any of the authors.

Open Access. This article is distributed under the terms of the Creative Commons Attribution-NonCommercial 4.0 International License (http://creativecommons.org/licenses/ by-nc/4.0/), which permits any noncommercial 
use, distribution, and reproduction in any medium, provided you give appropriate credit to the original author(s) and the source, provide a link to the Creative Commons license, and indicate if changes were made.

\section{REFERENCES}

1. Bays H. Sodium glucose co-transporter type 2 (SGLT2) inhibitors: targeting the kidney to improve glycemic control in diabetes mellitus. Diabetes Ther. 2013;4(2):195-220.

2. Kalra S. Sodium glucose co-transporter-2 (SGLT2) inhibitors: a review of their basic and clinical pharmacology. Diabetes Ther. 2014;5(2):355-66.

3. Sha S, Devineni D, Ghosh A, et al. Canagliflozin, a novel inhibitor of sodium glucose co-transporter 2 , dose dependently reduces calculated renal threshold for glucose excretion and increases urinary glucose excretion in healthy subjects. Diabetes Obes Metab. 2011;13(7):669-72.

4. Zanoli L, Granata A, Lentini $P$, et al. Sodium-glucose linked transporter-2 inhibitors in chronic kidney disease. Sci World J. 2015;2015:6. doi:10.1155/2015/317507.

5. Rosenwasser RF, Sultan S, Sutton D, Choksi R, Epstein BJ. SGLT-2 inhibitors and their potential in the treatment of diabetes. Diabetes Metab Syndr Obes. 2013;6:453-67.

6. Gerich J. Role of the kidney in normal glucose homeostasis and in the hyperglycaemia of diabetes mellitus: therapeutic implications. Diabetic Med. 2010;27(2):136-42.

7. Cherney DZ, Scholey JW, Jiang S, et al. The effect of direct renin inhibition alone and in combination with ACE inhibition on endothelial function, arterial stiffness, and renal function in type 1 diabetes. Diabetes Care. 2012;35(11):2324-30 (Epub 2012/07/28).

8. De Nicola L, Gabbai FB, Liberti ME, Sagliocca A, Conte G, Minutolo R. Sodium/glucose cotransporter 2 inhibitors and prevention of diabetic nephropathy: targeting the renal tubule in diabetes. Am J Kidney Dis. 2014;64(1):16-24.

9. Panchapakesan U, Pegg K, Gross S, et al. Effects of SGLT2 inhibition in human kidney proximal tubular cells-renoprotection in diabetic nephropathy? PLoS One. 2013;8(2):e54442.
10. Vallon V, Richter K, Blantz RC, Thomson S, Osswald $\mathrm{H}$. Glomerular hyperfiltration in experimental diabetes mellitus potential role of tubular reabsorption. J Am Soc Nephrol. 1999;10(12):2569-76.

11. Mather A, Pollock C. Glucose handling by the kidney. Kidney Int. 2011;79:S1-6.

12. Wright EM, Turk E. The sodium/glucose cotransport family SLC5. Pflügers Archiv. 2004;447(5):510-8.

13. Wright EM. Renal Na+-glucose cotransporters. Am J Physiol Renal Physiol. 2001;280(1):F10-8.

14. Abdul-Ghani M, DeFronzo R. Inhibition of renal glucose reabsorption: a novel strategy for achieving glucose control in type 2 diabetes mellitus. Endocr Pract. 2008;14(6):782-90.

15. DeFronzo R, Davidson J, Del Prato S. The role of the kidneys in glucose homeostasis: a new path towards normalizing glycaemia. Diabetes Obes Metab. 2012;14(1):5-14.

16. Komoroski B, Vachharajani N, Boulton D, et al. Dapagliflozin, a novel SGLT2 inhibitor, induces dose-dependent glucosuria in healthy subjects. Clin Pharmacol Ther. 2009;85(5):520-6.

17. Seman L, Macha S, Nehmiz G, et al. Empagliflozin (BI 10773), a potent and selective SGLT2 inhibitor, induces dose-dependent glucosuria in healthy subjects. Clin Pharmacol Drug Dev. 2013;2(2):152-61.

18. Grempler R, Thomas L, Klein T, et al. Weight loss induced by the potent and selective SGLT2 inhibitor, BI 10773, is due to body fat reduction. Studies in dietary-induced obese rats. Diabetes. In: Abstract 1793-P presented at the 70th Scientific Sessions of the American Diabetes Association, Orlando, FL, USA. 2010;59:469.

19. Bays HE, Weinstein R, Law G, Canovatchel W. Canagliflozin: effects in overweight and obese subjects without diabetes mellitus. Obesity. 2014;22(4):1042-9.

20. List JF, Whaley JM. Glucose dynamics and mechanistic implications of SGLT2 inhibitors in animals and humans. Kidney Int. 2011;79:S20-7.

21. Vasilakou D, Karagiannis T, Athanasiadou E, et al. Sodium-glucose cotransporter 2 inhibitors for type 2 diabetes: a systematic review and meta-analysis. Ann Intern Med. 2013;159(4):262-74.

22. Neumiller JJ. Empagliflozin: a new sodium-glucose co-transporter 2 (SGLT2) inhibitor for the treatment of type 2 diabetes. Drugs Context. 2014;3(212262):10.7573. 
23. Poole RM, Dungo RT. Ipragliflozin: first global approval. Drugs. 2014;74(5):611-7.

24. Nauck MA, Del Prato S, Meier JJ, et al. Dapagliflozin versus glipizide as add-on therapy in patients with Type 2 diabetes who have inadequate glycemic control with metformin a randomized, 52-week, double-blind, active-controlled noninferiority trial. Diabetes Care. 2011;34(9):2015-22.

25. Organisation CDsC. List of approved drug. http:// cdsco.nic.in/. Accessed Dec 24, 2015. 2015.

26. Bailey C, Iqbal N, T'joen C, List J. Dapagliflozin monotherapy in drug-naïve patients with diabetes: a randomized-controlled trial of low-dose range. Diabetes Obes Metab. 2012;14(10):951-9.

27. Cefalu WT, Leiter LA, Yoon K-H, et al. Efficacy and safety of canagliflozin versus glimepiride in patients with type 2 diabetes inadequately controlled with metformin (CANTATA-SU): 52 week results from a randomised, double-blind, phase 3 non-inferiority trial. The Lancet. 2013;382(9896):941-50.

28. Devineni D, Morrow L, Hompesch $\mathrm{M}$, et al. Canagliflozin improves glycaemic control over 28 days in subjects with type 2 diabetes not optimally controlled on insulin. Diabetes Obes Metab. 2012;14(6):539-45.

29. Ferrannini E, Seman L, Seewaldt-Becker E, Hantel S, Pinnetti S, Woerle H. A Phase IIb, randomized, placebo-controlled study of the SGLT2 inhibitor empagliflozin in patients with type 2 diabetes. Diabetes Obes Metab. 2013;15(8):721-8.

30. Stenlöf K, Cefalu W, Kim KA, et al. Efficacy and safety of canagliflozin monotherapy in subjects with type 2 diabetes mellitus inadequately controlled with diet and exercise. Diabetes Obes Metab. 2013;15(4):372-82.

31. Scheen AJ. Pharmacokinetics, Pharmacodynamics and clinical use of SGLT2 inhibitors in patients with Type 2 diabetes mellitus and chronic kidney disease. Clin Pharmacokinet. 2015;54(7):691-708.

32. Devineni D, Curtin CR, Marbury TC, et al. Effect of hepatic or renal impairment on the pharmacokinetics of canagliflozin, a sodium glucose co-transporter 2 inhibitor. Clin Ther. 2015;37(3):610-28. e4.

33. Kasichayanula S, Liu X, Pe Benito M, et al. The influence of kidney function on dapagliflozin exposure, metabolism and pharmacodynamics in healthy subjects and in patients with type 2 diabetes mellitus. $\mathrm{Br} \mathrm{J}$ Clin Pharmacol. 2013;76(3):432-44.
34. Kohan DE, Fioretto P, Tang W, List JF. Long-term study of patients with type 2 diabetes and moderate renal impairment shows that dapagliflozin reduces weight and blood pressure but does not improve glycemic control. Kidney Int. 2014;85(4):962-71.

35. Macha S, Mattheus M, Halabi A, Pinnetti S, Woerle $\mathrm{H}$, Broedl U. Pharmacokinetics, pharmacodynamics and safety of empagliflozin, a sodium glucose cotransporter 2 (SGLT2) inhibitor, in subjects with renal impairment. Diabetes Obes Metab. 2014;16(3):215-22.

36. Yale JF, Bakris G, Cariou B, et al. Efficacy and safety of canagliflozin in subjects with type 2 diabetes and chronic kidney disease. Diabetes Obes Metab. 2013;15(5):463-73.

37. Cherney DZ, Perkins BA, Soleymanlou N, et al. The renal hemodynamic effect of SGLT2 inhibition in patients with type 1 diabetes. Circulation. 2013:CIRCULATIONAHA. 113.005081.

38. Giunti S, Barit D, Cooper ME. Mechanisms of diabetic nephropathy role of hypertension. Hypertension. 2006;48(4):519-26.

39. Van Buren PN, Toto R. Hypertension in diabetic nephropathy: epidemiology, mechanisms, and management. Adv Chronic Kidney Dis. 2011;18(1):28-41.

40. Cherney DZ, Scholey JW, Miller J. Insights into the regulation of renal hemodynamic function in diabetic mellitus. Curr Diabetes Rev. 2008;4(4):280-90.

41. Sasson AN, Cherney D. Renal hyperfiltration related to diabetes mellitus and obesity in human disease. World J Diabetes. 2012;3(1):1-6.

42. Jerums G, Premaratne E, Panagiotopoulos S, MacIsaac R. The clinical significance of hyperfiltration in diabetes. Diabetologia. 2010;53(10):2093-104.

43. Melsom T, Mathisen UD, Ingebretsen OC, et al. Impaired fasting glucose is associated with renal hyperfiltration in the general population. Diabetes Care. 2011;34(7):1546-51.

44. Magee GM, Bilous R, Cardwell CR, Hunter SJ, Kee F, Fogarty DG. Is hyperfiltration associated with the future risk of developing diabetic nephropathy? A meta-analysis. Diabetologia. 2009;52(4):691-7.

45. Brenner BM, Cooper ME, de Zeeuw D, et al. Effects of losartan on renal and cardiovascular outcomes in patients with type 2 diabetes and nephropathy. N Engl J Med. 2001;345(12):861-9. 
46. De Azevedo M, Ramos O, Gross J. Lack of effect of captopril on glomerular hyperfiltration in normoalbuminuric normotensive insulin-dependent diabetic patients. Horm Metab Res. 1997;29(10):516-9.

47. Ficociello LH, Perkins BA, Silva KH, et al. Determinants of progression from microalbuminuria to proteinuria in patients who have type 1 diabetes and are treated with angiotensin-converting enzyme inhibitors. Clin J Am Soc Nephrol. 2007;2(3):461-9 Epub 2007/08/ 19.

48. Sochett EB, Cherney DZ, Curtis JR, Dekker MG, Scholey JW, Miller JA. Impact of renin angiotensin system modulation on the hyperfiltration state in type 1 diabetes. J Am Soc Nephrol. 2006;17(6):1703-9.

49. Ismail N, Becker B, Strzelczyk P, Ritz E. Renal disease and hypertension in non-insulin-dependent diabetes mellitus. Kidney Int. 1999;55(1):1-28 (Epub 1999/01/20).

50. Keller CK, Bergis KH, Fliser D, Ritz E. Renal findings in patients with short-term type 2 diabetes. J Am Soc Nephrol. 1996;7(12):2627-35 (Epub 1996/12/ 01).

51. Association AD. Standards of medical care in diabetes-2013. Diabetes Care. 2013;36(Suppl 1):S11.

52. Terami N, Ogawa D, Tachibana H, et al. Long-term treatment with the sodium glucose cotransporter 2 inhibitor, dapagliflozin, ameliorates glucose homeostasis and diabetic nephropathy in $\mathrm{db} / \mathrm{db}$ mice. PLoS One. 2014;9(6):e100777.

53. Gembardt F, Bartaun C, Jarzebska N, et al. The SGLT2 inhibitor empagliflozin ameliorates early features of diabetic nephropathy in BTBR ob/ob type 2 diabetic mice with and without hypertension. Am J Physiol Renal Physiol. 2014;307(3):F317-25.

54. Komala MG, Gross S, Mudaliar H, et al. Inhibition of kidney proximal tubular glucose reabsorption does not prevent against diabetic nephropathy in type 1 diabetic eNOS knockout mice. PLoS One. 2014;9(11):e108994.

55. Janssen Initiates CREDENCE Study in Patients with Type 2 Diabetes and Diabetic Nephropathy. http:// www.investor.jnj.com/releasedetail.cfm?ReleaseID= 827412. Accessed April 22, 2016.

56. Oliva RV, Bakris GL. Blood pressure effects of sodium-glucose co-transport 2 (SGLT2) inhibitors. J Am Soc Hypertens. 2014;8(5):330-9.
57. Weber MA, Mansfield TA, Iqbal N, Parikh SJ, Ptaszynska A. Dapagliflozin lowers HbA1c, systolic blood pressure and serum uric acid in patients with Type 2 diabetes and hypertension, regardless of class of concomitant antihypertensive therapy. Circulation. 2014;130(Suppl 2):A13640-A.

58. Lambers Heerspink H, De Zeeuw D, Wie L, Leslie B, List J. Dapagliflozin a glucose-regulating drug with diuretic properties in subjects with type 2 diabetes. Diabetes Obes Metab. 2013;15(9):853-62.

59. De Zeeuw D, Remuzzi G, Parving H-H, et al. Proteinuria, a target for renoprotection in patients with type 2 diabetic nephropathy: lessons from RENAAL. Kidney Int. 2004;65(6):2309-20.

60. Heerspink H, Johnsson E, Gause-Nilsson I, Cain V, Sjöström C. Dapagliflozin reduces albuminuria in patients with diabetes and hypertension receiving renin-angiotensin blockers. Diabetes Obes Metab. 2016;18(6):590-7.

61. Barnett AH, Mithal A, Manassie J, et al. Efficacy and safety of empagliflozin added to existing antidiabetes treatment in patients with type 2 diabetes and chronic kidney disease: a randomised, double-blind, placebo-controlled trial. Lancet Diabetes Endocrinol. 2014;2(5):369-84.

62. Chonchol M, Shlipak MG, Katz R, et al. Relationship of uric acid with progression of kidney disease. Am J Kidney Dis. 2007;50(2):239-47.

63. Goicoechea M, de Vinuesa SG, Verdalles U, et al. Effect of allopurinol in chronic kidney disease progression and cardiovascular risk. Clin J Am Soc Nephrol. 2010;5(8):1388-93.

64. Iseki K, Oshiro S, Tozawa M, Iseki C, Ikemiya Y, Takishita S. Significance of hyperuricemia on the early detection of renal failure in a cohort of screened subjects. Hypertens Res. 2001;24(6):691-7.

65. Hovind $\mathrm{P}$, Rossing $\mathrm{P}$, Johnson RJ, Parving $\mathrm{H}-\mathrm{H}$. Serum uric acid as a new player in the development of diabetic nephropathy. J Ren Nutr. 2011;21(1):124-7.

66. Kang D-H, Nakagawa T, Feng L, et al. A role for uric acid in the progression of renal disease. J Am Soc Nephrol. 2002;13(12):2888-97.

67. Bjornstad P, Lanaspa MA, Ishimoto T, et al. Fructose and uric acid in diabetic nephropathy. Diabetologia. 2015;58(9):1993-2002.

68. Bailey CJ, Gross JL, Pieters A, Bastien A, List JF. Effect of dapagliflozin in patients with type 2 diabetes who have inadequate glycaemic control with metformin: a randomised, double-blind, 
placebo-controlled trial. Lancet. 2010;375(9733): 2223-33.

69. Wilding J, Ferrannini E, Fonseca V, Wilpshaar W, Dhanjal P, Houzer A. Efficacy and safety of ipragliflozin in patients with type 2 diabetes inadequately controlled on metformin: a dose-finding study. Diabetes Obes Metab. 2013;15(5):403-9.

70. Usiskin K, Kline I, Fung A, Mayer C, Meininger G. Safety and tolerability of canagliflozin in patients with type 2 diabetes mellitus: pooled analysis of phase 3 study results. Postgrad Med. 2014;126(3):16-34.

71. Leiter LA, Yoon K-H, Arias P, et al. Canagliflozin provides durable glycemic improvements and body weight reduction over 104 weeks versus glimepiride in patients with type 2 diabetes on metformin: a randomized, double-blind, phase 3 study. Diabetes Care. 2015;38(3):355-64.

72. Goldin A, Beckman JA, Schmidt AM, Creager MA. Advanced glycation end products sparking the development of diabetic vascular injury. Circulation. 2006;114(6):597-605.

73. Hsieh C-L, Yang M-H, Chyau C-C, et al. Kinetic analysis on the sensitivity of glucose-or glyoxal-induced LDL glycation to the inhibitory effect of Psidium guajava extract in a physiomimic system. Biosystems. 2007;88(1):92-100.

74. Negre-Salvayre A, Salvayre R, Augé N, Pamplona R, Portero-Otin M. Hyperglycemia and glycation in diabetic complications. Antioxid Redox Signal. 2009;11(12):3071-109.

75. Maeda S, Matsui T, Takeuchi M. Yamagishi Si. Sodium-glucose cotransporter 2-mediated oxidative stress augments advanced glycation end products-induced tubular cell apoptosis. Diabetes Metab Res Rev. 2013;29(5):406-12.

76. Kojima N, Williams JM, Takahashi T, Miyata N, Roman RJ. Effects of a new SGLT2 inhibitor, luseogliflozin, on diabetic nephropathy in T2DN rats. J Pharm Exp Ther. 2013;345(3):464-72.

77. Zinman B, Wanner C, Lachin JM, et al. Empagliflozin, cardiovascular outcomes, and mortality in type 2 diabetes. $\mathrm{N}$ Engl J Med. 2015;373(22):2117-28.

78. Schernthaner G, Schernthaner-Reiter $\mathrm{MH}$, Schernthaner GH. EMPA-REG and other cardiovascular outcome trials of glucose-lowering agents: implications for future treatment strategies in Type 2 diabetes mellitus. Clin Ther. 2016;38(6):1288-98.

79. Nauck MA. Update on developments with SGLT2 inhibitors in the management of type 2 diabetes. Drug Des Dev Ther. 2014;8:1335.

80. Shawwa K EF, Samaha A. A case of Fanconi syndrome in a patient taking canagliflozin for diabetes: a previously unreported catastrophic adverse effect. Am J Kidney Dis 67(5):A99.

81. K/DOQI clinical practice guidelines for chronic kidney disease. evaluation, classification, and stratification. Am J Kidney Dis. 2002;39(2 Suppl 1):S1-266 (Epub 2002/03/21).

82. (Invokana ${ }^{\mathrm{TM}}$ ) C. Prescribing Information. Titusville, NJ: Janssen Pharmaceuticals, Inc. http:// www.accessdata.fda.gov/drugsatfda_docs/label/2013/ 204042s000lbl.pdf. On April 22, 2016.

83. (JardianceTM) E. Prescribing information. Ridgefield, CT: Boehringer Ingelheim Pharmaceuticals, Inc. http://bidocs.boehringeringelheim.com/BIWebAccess/ViewServlet.ser?doc Base=renetnt\&folderPath=/Prescribing+Information/ PIs/Jardiance/jardiance.pdf. Accessed April 15, 2016.

84. Dapagliflozin (Farxiga ${ }^{\mathrm{TM}}$ ) Prescribing information. Wilmington DAhwa-ucppfppAoD, 2015. Prescribing information. Wilmington, Delaware: AstraZeneca. http://www1.astrazeneca-us.com/pi/ pi_farxiga.pdf\#page=1. Accessed April 22, 2016. 